\title{
FAST PHASE-DIFFERENCE-BASED DOA ESTIMATION USING RANDOM FERNS
}

\author{
Hui Chen, Tarig Ballal and Tareq Y. Al-Naffouri \\ Computer, Electrical and Mathematical Sciences \& Engineering Division \\ King Abdullah University of Science and Technology (KAUST), Thuwal, 23955-6900
}

\begin{abstract}
Direction of arrival (DOA) information of a signal is important in communications, localization, object tracking and so on. Frequency-domain-based time-delay estimation is capable of achieving DOA in subsample accuracy; however, it suffers from the phase wrapping problem. In this paper, a frequency-diversity based method is proposed to overcome the phase wrapping problem. Inspired by the machine learning technique of random ferns, an algorithm is proposed to speed up the search procedure. The performance of the algorithm is evaluated based on three different signal models using both simulations and experimental tests. The results show that using random ferns can reduce search time to $1 / 6$ of the search time of the exhaustive method while maintaining the same accuracy. The proposed search approach outperforms a benchmark frequency-diversity based algorithm by offering lower DOA estimation error.
\end{abstract}

Index Terms - Direction of Arrival, Random Ferns, Machine Learning, Ultrasound, Phase-difference

\section{INTRODUCTION}

Knowing the direction of a target is essential in many applications such as wireless sensor networks localization [1], communications [2], acoustic event detection [3], indoor localization [4], hand gesture recognition [5] and so on.

DOA estimation methods aim to estimate the direction of arrival of a signal observed by multiple spatially separated receivers. A summary of key DOA estimators can be found in [6]. Time delay based DOA estimation [7] is one of the widely used approaches. Time delay can be measured in the frequency-domain or time-domain. Frequency-based time-delay estimation has the advantage of providing subsample time-delay resolution [8] [9]. However, the phase wrapping problem will appear if the receiver separation is large. This problem can be solved by spatial-diversity based algorithms [10] leveraging observations from several sensors, or frequency-diversity based algorithms [8] using wide signal bandwidth.

In this paper, we consider a single-source scenario where the goal is to estimate 1-D angle with a pair of sensors. This setup can easily be extended to the 2-D DOA estima- tion case by employing one or more non-collinear receivers. To estimate DOA, an exhaustive grid search method is proposed. The proposed method aims to find the source direction that minimizes the mismatch between the observed (usually wrapped) phase difference and the phase difference calculated based on hypothesized source directions. In order to reduce the computational complexity and speed up the search operation, we leverage tools from machine learning by treating the DOA estimation as a pattern recognition problem. Namely, we propose a DOA estimation algorithm based on the classification method of random ferns [11][12].The proposed algorithm aims to find an estimate of the source direction in a rapid manner by utilizing the structure of the mismatch as a function of the hypothesized source direction.

The contributions of this work are: a) A grid search method for multi-frequency DOA estimation that solves the phase unwrapping problem; b) A fast algorithm that implements the search process by employing random ferns; c) Comprehensive performance evaluation from both simulations and experimental tests using three types of signals. The code of the algorithm can be found in DOA Algorithms.

This paper is organized as follows. Section II states the DOA estimation problem using multi-frequency phasedifference and explains how to speed up the grid-search method using the idea of random ferns. Section III describes the signal models and the experiment setup, and discusses the simulation and experimental results. In section $\mathrm{V}$ we derive the conclusions of the whole work with suggestions of future directions.

\section{DIRECTION-OF-ARRIVAL ESTIMATION USING RANDOM FERNS}

\subsection{Frequency-Diversity-Based DOA Algorithm}

The far-field model for estimating the DOA of a signal received by a pair of sensors with separation $D$ is shown in Fig. 1. For a certain angle $\theta$, the noise-free phase difference vector $\phi_{i}$ at frequency components $f_{i}$ with wavelength $\lambda_{i}$ can be calculated as

$$
\phi_{i}(\theta)=\frac{2 \pi d}{\lambda_{i}}=\frac{2 \pi f_{i} \sin (\theta) D}{v}(i=1, \ldots, N),
$$

where $v$ is the speed of signal propagation. 
The observed phase-difference can be calculated from the signals received at a pair of sensors $S_{1}$ and $S_{2}$ (see Fig. 1) as [5]

$$
\hat{\psi}_{i}=\operatorname{ang}\left(Y_{1}\left(f_{i}\right) \cdot Y_{2}^{*}\left(f_{i}\right)\right)=\hat{\phi}_{i}-2 \pi N_{i},
$$

where $Y_{1}$ and $Y_{2}$ are the DFT (Discrete Fourier Transform) of the received signals at sensor $S_{1}$ and sensor $S_{2}$ respectively, $(\cdot)^{*}$ indicates the complex conjugate operation, $\hat{\phi}_{i}$ is the noisy unwrapped phase difference, and $N_{i}$ is an integer.

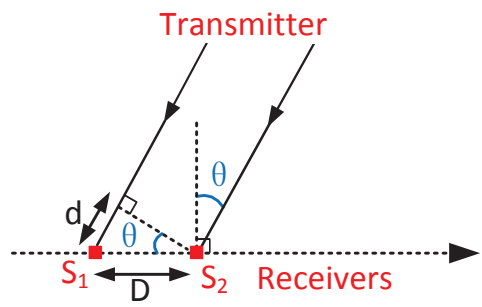

Fig. 1. Far-field model.

The proposed grid search method is based on the notion that the set of observed (wrapped) phase difference values $\psi_{i}, i=1, \cdots, N$ for $N>1$ is unique for each DOA. A sufficient condition for uniqueness is given in [8]. Assuming that the frequencies $f_{i}$ satisfy the uniqueness criterion, we form the phase-difference mismatch function for a hypothesized source direction $\theta_{m}$ as

$$
e\left(\theta_{m}\right)=\sum_{i=1}^{N}\left|\hat{\psi}_{i}-\psi_{i}\left(\theta_{m}\right)\right|=\sum_{i=1}^{N}\left|\hat{\psi}_{i}-\operatorname{wrap}\left(\phi_{i}\left(\theta_{m}\right)\right)\right|,
$$

where $\phi_{i}\left(\theta_{m}\right)$ is the phase difference at frequency $f_{i}$ corresponding to $\theta_{m}$ calculated using Equation (1), $\operatorname{wrap}($.$) is the$ operation which limits the difference within $(-\pi, \pi]$ as

$$
\operatorname{wrap}(\alpha)=\left(\frac{\alpha}{2 \pi}-\left\lfloor\frac{\alpha}{2 \pi}\right\rceil\right) \cdot 2 \pi,
$$

where $L$.$\rceil returns the nearest integer. By evaluating Equa-$ tion (3) for a grid of $M$ values $\left(\theta_{m}\right)$ covering the whole field of view, we can estimate the DOA as the one with the phase differences that produce the smallest mismatch with the observations. Namely,

$$
\hat{\theta}=\arg \min _{\theta_{m}}\left\{e\left(\theta_{m}\right)\right\} .
$$

Using Equation (3), we can predict the mismatch error for a certain source direction $\theta$ by replacing $\hat{\psi}_{i}$ with a calculated $\hat{\psi}_{i}(\theta)$. The resultant mismatch function calculated using $\hat{\psi}_{i}(\theta)$ will, more or less, follow the same pattern of the mismatch function calculated based on noisy observations. This is due to the fact that in reasonable noise conditions, the general structure of the mismatch function is dominated by the effect of phase wrapping at different frequencies. The mismatch function (3) is evaluated for $\hat{\psi}_{i}=\hat{\psi}_{i}(\theta)$ for different choices of $\theta$. The results for $\theta=0^{\circ}, 15^{\circ}, 30^{\circ}, 45^{\circ}$ with $N=4$ are shown in Fig. 2. It can be seen from the figure that different $\theta$ values have different error patterns, and this provides the possibility of solving angle estimation as a pattern recognition problem. Subsequently, we will explain the random ferns algorithm and apply it as a fast and less complex alternative to find the true source direction.

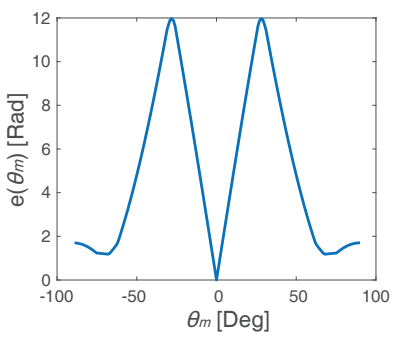

(a) $\theta=0^{\circ}$

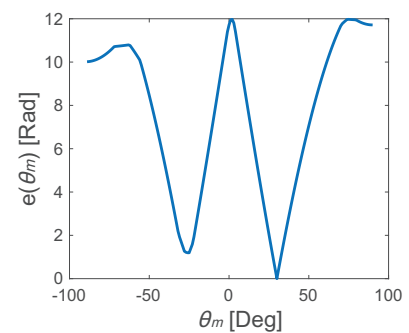

(c) $\theta=30^{\circ}$

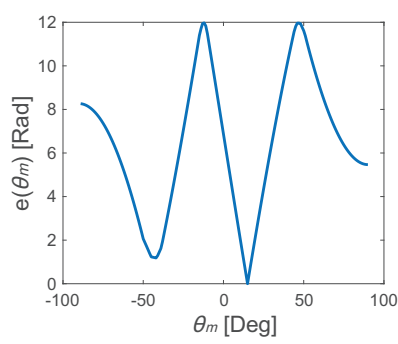

(b) $\theta=15^{\circ}$

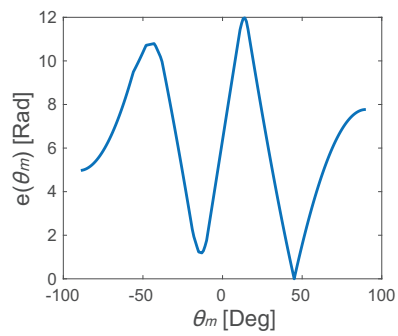

(d) $\theta=45^{\circ}$
Fig. 2. The mismatch function for different source directions.

\subsection{Random Ferns and Fern Matrix}

Random ferns [11] is an algorithm firstly proposed for image classification. Random ferns take a set of features and create a histogram of the training data from the same class. This helps the machine to do image classification using only a few number of pixel values. More details on this topic can be found in [11]. Inspired by this idea, we start by creating an error matrix $\boldsymbol{E}$ utilizing Equation (3)

$$
E_{m 1, m 2}=\sum_{i=1}^{N}\left|\operatorname{wrap}\left[\phi_{i}\left(\theta_{m 1}\right)-\phi_{i}\left(\theta_{m 2}\right)\right]\right|,
$$

where $E_{m 1, m 2}$ is the element in row $m 1$, column $m 2$ of the matrix $\boldsymbol{E}, m 1, m 2 \in[0, M]$ and hence the error matrix has a size of $M \times M$.

The next step is creating a fern matrix $\boldsymbol{R}$ from the error matrix $\boldsymbol{E}$. By randomly picking $k$ angles from the $M$ candidates as fern points, a set of $K=(k$ choose 2$)=k(k-1) / 2$ pairs can be created. The fern vector $\boldsymbol{r}_{m}$ for the $m^{t h}$ candidate can be calculated as

$$
r_{m, i}=\left\{\begin{aligned}
1, & E_{m, K_{i, 1}} \geq E_{m, K_{i, 2}} \\
-1, & E_{m, K_{i, 1}}<E_{m, K_{i, 2}}
\end{aligned}\right.
$$


where $r_{m, i}$ is the $i^{\text {th }}$ element of the vector $\boldsymbol{r}_{m}, K_{i, 1}$ and $K_{i, 2}$ are the first and second element of the $i^{t h}$ pair. $\boldsymbol{r}_{m}$ is the $m^{t h}$ row of the fern matrix $\boldsymbol{R}$ which has the size of $M \times K$.

An example of encoding error pattern into a fern vector at $10^{\circ}$ is shown in Fig. 3. $k=5$ is chosen in this case, and the fern points are $\left[-69^{\circ},-29^{\circ},-4^{\circ}, 7^{\circ}, 47^{\circ}\right]$. The encoded fern vector for the pair $(7,47),(-4,47),(-4,7), \ldots,(-69,-29)$ is $[-1,-1,1, \ldots,-1]^{T}$ using Equation (7).

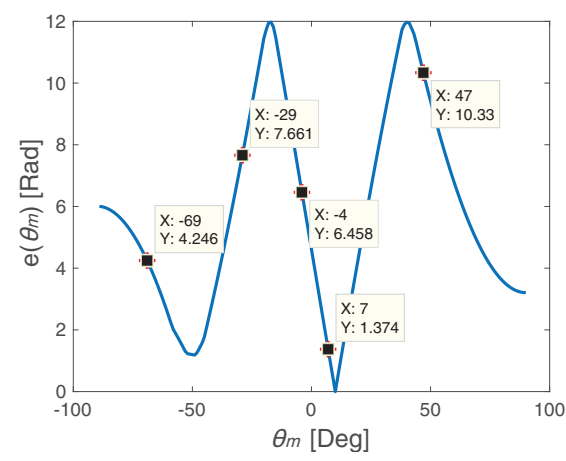

Fig. 3. An example of encoding a fern vector.

\subsection{Algorithm for DOA Estimation Using Random Ferns}

For any phase difference vector obtained from the received signal as per Equation (2), the error at each fern point can be calculated and hence the fern vector $\boldsymbol{r}_{\text {est }}$ can be calculated from Equation (7). We can then reduce the search area by finding the highest cross-correlation value in $c$ given by

$$
\boldsymbol{c}=\boldsymbol{R} \boldsymbol{r}_{\text {est }} .
$$

The DOA estimation algorithm using random ferns can be summarized as follows: 1-Calculate the errors at fern points using Equation (3); 2-Encode the received signal into fern vector $r_{\text {est }}$ using Equation (7); 3-Obtain the candidate crosscorrelation values using Equation (8). 4-Choose the candidates with the highest values (Some candidates may have the same fern vector which results in the same cross-correlation value). 5-Find the least phase difference error among the DOA candidates using Equation (5) to obtain the search result.

By encoding the fern vector from binary to decimal, the DOA candidates can be clustered into several groups as shown in Fig. 4. It should be noted that the combined size of these groups is much smaller than the maximum index which has the value of $2^{K}$.

The processing time for the exhaustive search algorithm can be expressed as

$$
T_{\text {full }}=\frac{\gamma}{\delta} t_{1}
$$

where $t_{1}$ is the the processing time for each sample, $\gamma$ is the search range and $\delta$ is the search angle resolution. The time

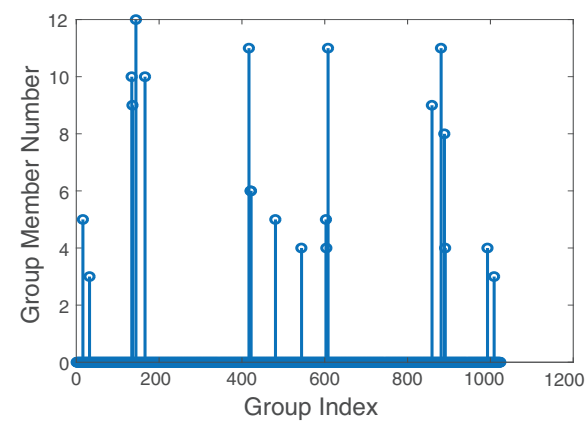

Fig. 4. Clustered angle candidates.

for using random ferns can be expressed as

$$
T_{\text {fern }}=\frac{\gamma}{\delta g} t_{1}+t_{2}
$$

where $t_{2}$ is the time for fern matrix multiplication, $g$ is the number of groups obtained from the fern vector.

\subsection{Fern Points Selection}

Randomized fern points selection will cause uncertainty and unpredictable performance. Consecutive points, for example, may not classify the candidates appropriately. Hence, it is necessary to have a criterion to evaluate the selection of fern points. We will use entropy as the indicator for evaluating the Fern points selection. For a fern matrix with size $M \times K$, the angles with the same code will be grouped together to form $L$ groups. The entropy $H$ can be calculated as

$$
H=-\sum_{i=1}^{L} p_{i} \log \left(p_{i}\right)
$$

where $p_{i}$ is the number of candidates in group $i$ divided by $M$. The entropy $H$ is higher for larger fern vector sizes. For a certain fern vector length, a high entropy value indicates a good choice of fern angles. The fern angles selection procedure can be performed over a large number of simulation trials and the points with the highest entropy are chosen. The effect of different fern vector length will be discussed in Section III.

\section{SIMULATION AND EXPERIMENTAL TESTS}

\subsection{Signal Models}

We consider three different multi-frequency signal models. OFH (Orthogonal Frequency Hops) signal hops between a set of carrier frequencies and it is widely used in communication and ranging due to its robustness to multipath and noise [13]. The design of OFS (Orthogonal Frequency Sum) signal is similar to OFDM [14] which combines sinusoidal signals with different carrier frequencies. ZC (Zadoff-Chu) sequence is a polyphase complex-valued zero-autocorrelation 
sequence [15] and the design of the signal can be found in [16].

The proposed algorithm is tested on OFH, OFS and ZC signals. In order to make a fair comparison between signals, we choose the parameter to make sure that the different signals have the same duration $(4.1 \mathrm{~ms}$ followed by a $12.6 \mathrm{~ms}$ silent period), bandwidth ( $19 \mathrm{kHz}$ to $23 \mathrm{kHz}$ ) and energy.

\subsection{Simulations}

\subsubsection{Fern Points Selection}

In simulation, fern points number $k$ from 2 to 7 were tested. In each case, $k$ fern points are chosen randomly for 1000 times and the one with the highest entropy is recorded in Table 1 with max group size (Max GS), as well as average group size (Avg GS) information. The processing time (the average processing time for one measurement) is compared with the $1.129 \mathrm{~ms}$ of the full-range-search algorithm. In order to save computational resources while maintaining processing time, $k=5$ will be chosen for the real test.

Table 1. Fern Points Selection Summary

\begin{tabular}{c|ccccc}
\hline $\begin{array}{c}\text { Points } \\
\text { Num }\end{array}$ & $\begin{array}{c}\text { Fern } \\
\text { Bits }\end{array}$ & $\begin{array}{c}\text { Max } \\
\text { Entropy }\end{array}$ & $\begin{array}{c}\text { Max } \\
\text { GS }\end{array}$ & $\begin{array}{c}\text { Avg } \\
\text { GS }\end{array}$ & $\begin{array}{c}\text { Processing } \\
\text { Time }[\mathrm{ms}]\end{array}$ \\
\hline 2 & 1 & 1.000 & 90 & 90 & 0.728 \\
\hline 3 & 3 & 2.496 & 47 & 30 & 0.301 \\
\hline 4 & 6 & 3.507 & 23 & 12.86 & 0.203 \\
\hline 5 & 10 & 4.218 & 16 & 6.92 & 0.174 \\
\hline 6 & 15 & 4.996 & 14 & 4.09 & 0.180 \\
\hline 7 & 21 & 5.397 & 11 & 3.11 & 0.189 \\
\hline
\end{tabular}

\subsubsection{Simulation Results}

Fig. 5 (a) plots the RMSE (root-mean-squared-error) of DOA estimation versus signal-to-noise ratio (SNR) for DOA of $0^{\circ}$, while Fig. 5 (b) plots the RMSE for different DOA choices at $\mathrm{SNR}=5 \mathrm{~dB}$. In both cases, and for each signal type, the proposed method clearly outperform the benchmark method [8].

\subsection{Experiments}

\subsubsection{Experimental Setup}

The experimental tests were carried out in a typical room environment with indoor temperature of $24^{\circ} \mathrm{C}$. The distance between the two receivers is $1.8 \mathrm{~cm}$. The transmitter and the receivers were placed 1.5 meters above the ground with 2 meters distance. The transmitter was located at 6 different DOAs with fixed SNR. The ground truth is obtained by the ARTTRACK5 [17] infrared optical 6-DOF (Degree of Freedom) system with passive markers. This system has the positioning resolution of $0.1 \mathrm{~mm}$ and the update rate of $300 \mathrm{~Hz}$.

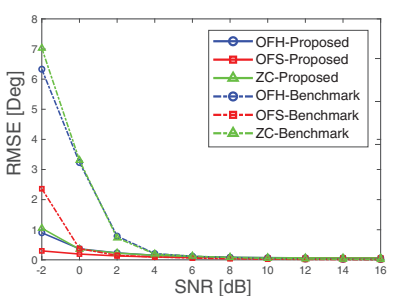

(a) SNR vs. RMSE $\left(0^{\circ}\right)$

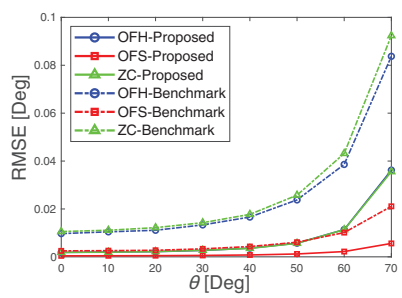

(b) RMSE for different DOAs
Fig. 5. Simulation results.

\subsubsection{Experimental Results}

The experimental results are affected by different factors such as multipath effect, calibration errors, etc. These effects might give rise to outliers and other phenomena that are not present in the simulation results. Therefore, a meaningful performance evaluation that limits the impact of these effects is found to be the percentage of cases in which the estimation exhibits error below a certain level. Two degrees of error is chosen as the benchmark point.

Fig. 6 plots the performance of the proposed method and benchmark method for different DOA choices. The results are obtained from 500 experimental evaluations at each DOA with the SNR of $0 d B$. The proposed method clearly outperforms the benchmark method [8] over all the signal types.

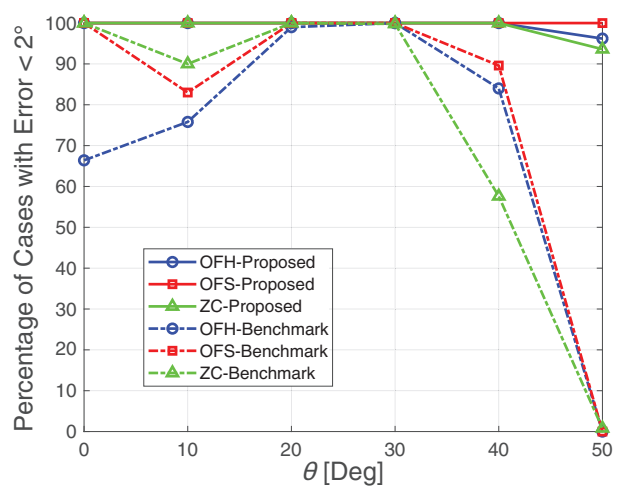

Fig. 6. Experimental results for source at different DOAs.

\section{CONCLUSION}

An algorithm for DOA estimation from phase-difference observations is presented. The proposed algorithm applies the machine learning approach of random ferns to speed up computations by 6 times compared to exhaustive search while maintaining the same accuracy. The proposed algorithm is tested using three different types. Simulation and experimental results confirm the effectiveness of the proposed approach. Future works will focus on studying the effect of Doppler, multipath and multi-user interference. 


\section{REFERENCES}

[1] Peng Rong and Mihail L Sichitiu, "Angle of arrival localization for wireless sensor networks," in Sensor and Ad Hoc Communications and Networks, 2006. SECON'06. 2006 3rd Annual IEEE Communications Society on. IEEE, 2006, vol. 1, pp. 374-382.

[2] Lal C Godara, "Application of antenna arrays to mobile communications. ii. beam-forming and directionof-arrival considerations," Proceedings of the IEEE, vol. 85, no. 8, pp. 1195-1245, 1997.

[3] Yukang Guo and Mike Hazas, "Localising speech, footsteps and other sounds using resource-constrained devices," in Information Processing in Sensor Networks (IPSN), 2011 10th International Conference on. IEEE, 2011, pp. 330-341.

[4] Mateusz Rzymowski, P Woznica, and L Kulas, "Singleanchor indoor localization using espar antenna," IEEE Antennas and Wireless Propagation Letters, vol. 15, pp. 1183-1186, 2016.

[5] Hui Chen, Tarig Ballal, Mohamed Saad, and Tareq Y Al-Naffouri, "Angle-of-arrival-based gesture recognition using ultrasonic multi-frequency signals," in Signal Processing Conference (EUSIPCO), 2017 25th European. IEEE, 2017, pp. 16-20.

[6] Hamid Krim and Mats Viberg, "Two decades of array signal processing research: the parametric approach," IEEE signal processing magazine, vol. 13, no. 4, pp. 6794, 1996.

[7] A Quazi, "An overview on the time delay estimate in active and passive systems for target localization," IEEE Transactions on Acoustics, Speech, and Signal Processing, vol. 29, no. 3, pp. 527-533, 1981.

[8] Tarig Ballal and Chris J Bleakley, "Doa estimation for a multi-frequency signal using widely-spaced sensors," in Signal Processing Conference, 2010 18th European. IEEE, 2010, pp. 691-695.

[9] Tarig Ballal and Chris J Bleakley, "Phase-difference ambiguity resolution for a single-frequency signal," IEEE Signal Processing Letters, vol. 15, pp. 853-856, 2008.

[10] Tarig Ballal and Chris J Bleakley, "Doa estimation of multiple sparse sources using three widely-spaced sensors," in Signal Processing Conference, 2009 17th European. IEEE, 2009, pp. 1978-1982.

[11] Anna Bosch, Andrew Zisserman, and Xavier Munoz, "Image classification using random forests and ferns," in Computer Vision, 2007. ICCV 2007. IEEE 11th International Conference on. IEEE, 2007, pp. 1-8.
[12] Mustafa Ozuysal, Michael Calonder, Vincent Lepetit, and Pascal Fua, "Fast keypoint recognition using random ferns," IEEE transactions on pattern analysis and machine intelligence, vol. 32, no. 3, pp. 448-461, 2010.

[13] Mohamed M Saad, Chris J Bleakley, and Simon Dobson, "Robust high-accuracy ultrasonic range measurement system," IEEE Transactions on Instrumentation and Measurement, vol. 60, no. 10, pp. 3334-3341, 2011.

[14] Marian Oziewicz, "On application of music algorithm to time delay estimation in ofdm channels," IEEE Transactions on Broadcasting, vol. 51, no. 2, pp. 249-255, 2005.

[15] David Chu, "Polyphase codes with good periodic correlation properties (corresp.)," IEEE Transactions on information theory, vol. 18, no. 4, pp. 531-532, 1972.

[16] Mohammed H AlSharif, Mohamed Saad, Mohamed Siala, Tarig Ballal, Hatem Boujemaa, and Tareq Y AlNaffouri, "Zadoff-chu coded ultrasonic signal for accurate range estimation," in Signal Processing Conference (EUSIPCO), 2017 25th European. IEEE, 2017, pp. $1250-1254$.

[17] "Arttrack5 (advanced realtime tracking) technical data," https://ar-tracking.com/products / tracking-systems/arttrack5/, Accessed: 2018-06-28. 